\title{
Local analgesia after arthroplasties first use of absorbable compress report.
}

\author{
Dominique Persoons* \\ Hopital de Saverne, Cote de Saverne, France
}

\begin{abstract}
Background: Joint Replacement Surgery is subject to budget constraints, including shortened length of stay Red River Athletic Conference (RRAC). Local analgesia facilitates rapid postoperative recovery. Local soft tissue infiltration with Naropein ${ }^{\circledR}$ is recommended after joint replacement.
\end{abstract}

Methodology: Soft tissue infiltration around the operative site is limited by low efficiency due to rapid resorption. A haemostatic compress was imbibed with Naropein ${ }^{\circledR}$ and deposited near the operative site. The objective was to allow the gradual diffusion of the molecule until disapproval of this compress, about 3 days. The use of cellulose pad soaked with Naropein ${ }^{\circledR}$ may be an improvement over the simple local infiltration. No wound drainage was performed.

Result: The short prospective series aimed to demonstrate the effectiveness of postoperative anesthesia and the safety of the cellulose pad. This has been demonstrated along this preliminary study.

Conclusions: A short preliminary series suggests that infiltration of Naropein ${ }^{\circledR}$ into soft tissues may be replaced by the deposition of a Naropeine-soaked cellulose pad.

Keywords: Analgesia, Naropein, Cellulose pad, Naropein ${ }$, Surgery, Post-operative pain, Catheter, Local analgesia.

Accepted on September 24, 2018

\section{Introduction}

The use of Naropein $₫$ in the form of muscle wall infiltration following arthroplastic surgery is recognized [1]. Post-operative pain is considered to be generally severe in $60 \%$ of patients [2]. However, the simple bolus of intramuscular anesthetic drug gives analgesia less than $24 \mathrm{~h} \mathrm{[3],} \mathrm{which} \mathrm{is} \mathrm{considered}$ insufficient. The interest of single injection is limited by the intensity and duration of the post-operative pain. In addition, the interest of massive injection into a large scar during abdominal or gynecological surgery remains controversial. In orthopedic surgery, a similar debate is open.

In contrast, perineural catheters significantly reduced postoperative pain $[4,5]$ without local damage. They are officially the recommended analgesia in orthopedic surgery today. But neural toxicity of Naropein ${ }^{\circledR}$ and local analgesics is largely described.

In those conditions, local continuous infiltration seemed relevant. Placing a catheter inside the operative scar could be considered. Originally, this was intended to provide continuous administration of local anesthetic without risk of nerve damage. This idea extended the field of local analgesia to hip, knee and shoulder arthroplasty. A local catheter with various painkillers made the first postoperative days more comfortable and allowed for a very short hospital stay, with subsequent cost reduction $[6,7]$. Of course, in this case, drainage of the operating site is not allowed. While the recommendations point to a lack of drainage in hip or knee surgery [8]. But placing a catheter inside a surgical wound and injecting a pressure fluid did not seem as simple as expected. Many problems have been described with respect to the local administration of pressurized fluid [9].

\section{Material}

Contrary to a general consensus, our clinical experience has shown that the maintenance of perineural catheters, or even local catheters, in the surgical areas of the knee and hip prosthesis caused many problems, such as mismatching, twisting, or obstruction of the Naropein ${ }^{\circledR}$ bottle-feeding pouch. In the case of an early home return, perineural catheter monitoring was tedious or impossible. This situation was even considered dangerous because the catheter was placed near the operative site of the prosthesis.

We considered it was necessary to find a simpler local anesthesia method that would guarantee a prolonged release of at least $48 \mathrm{~h}$ and a lack of supervision by highly specialized staff.

We imagined replacing the local intramuscular catheter with a simple cellulose pad soaked with $150 \mathrm{mg}$ Naropein ${ }^{\circledR}$ and eventually $10 \mathrm{mg}$ morphine. The cellulose compress serves as a support for the progressive release of the anesthetic drug.

\section{Method}

Between January 2018 and May 2018, 60 patients had a hip or a knee arthroplasty (Table 1). They benefited from the

Table 1: Demographic Data for Patients in this Study

\begin{tabular}{|c|c|}
\hline Characteristics & Data \\
\hline Average Age & 67 years old \\
\hline Sex & $36 \mathrm{~W} / 24 \mathrm{M}$ \\
\hline Average Body Mass Index & $32.7 \pm 7.3$ \\
\hline $\begin{array}{c}\text { Average Stay in the Hospital (52 Patients) } \\
\text { Day Hospital (8 Patients) }\end{array}$ & 5.2 days \\
\hline Complication of the Wound - Immediate \\
Delaided & 0 \\
\hline Average Level of Pain at Discharge from Hospital & $3.5(2-5)$ \\
\hline
\end{tabular}




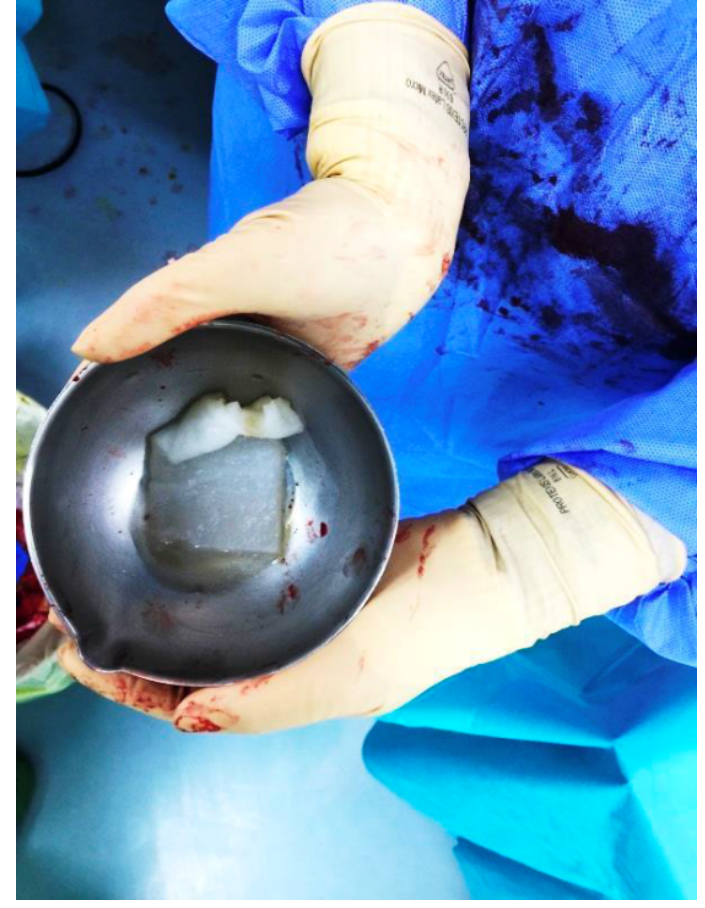

Figure 1: A fibrillated cellulose pad absorbs the analgesic in the water solution.

implantation of an oxidized cellulose pad impregnated with Naropeine ${ }^{\circledR}+/$ - morphine (Figure 1).

Exclusion criteria: Cellulose intolerance, Naropein ${ }^{\circledR}$ allergy, cardiovascular desease, poor general condition, liver failure, revision arthroplasties in relation to infection. 11 patients were excluded.

The continuous series was performed by a single operator. The other two surgeons in the department did not use this method, but retained the classic perioperative intramuscular infiltration. No perineural catheters were used during this period. In those conditions, the comparison between the two methods was easy.

Intuitive comparison with patients treated with intramuscular infiltration showed no inconvenience in patients treated with Naropein ${ }^{\circledR}$ pad. On the day of departure, no difference between the two groups was highlighted. Of these 60 patients, 9 chose a day hospitalization. They returned home at the evening of the intervention. 8 reported "normal" operative follow-up with pain less than or equal to 3 in 10 on the first postoperative day. Only one hip replacement patient reported pain at level 7 on 10 scale, during an all week. Her situation required 2 visits from her family doctor and is considered a failure of outpatient hospitalization.

Two data were studied:

- Tolerance: Is the oxidized cellulose compress well tolerated?

- Efficacy: Are the results with $150 \mathrm{mg}$ Naropein ${ }^{\circledR}$ pad at least comparable to the intramuscular series?

a) Tolerance:

Of 60 patients, 59 had a normal postoperative period. One

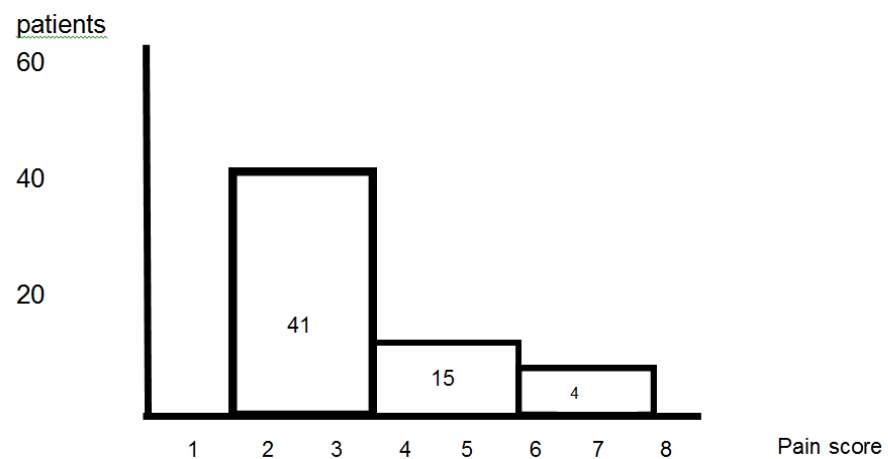

Figure 2: Iimmediate post-operative score at day +1 .

patient was readmitted late on the $21^{\text {st }}$ postoperative day with multiple leg ulcers. It was reoperated and no infection of the operated joint was observed. The compress had been gone for a long time. Staphylococcus aureus has been found inside superficial ulcers. The other 59 patients had no complications.

\section{b) Efficiency:}

The 60 patients had an immediate postoperative pain between 3 and 7 on a scale of 10 . Two thirds of the patients had a pain score of 3 from the first day (41 patients). 15\% (10 patients) had pain assessed at 6 on the first day and 4 on the day of delivery ( $3^{\text {rd }}$ to $5^{\text {th }}$ day) (Figure 2 ).

\section{Discussion}

Since the 1970s, the management of post-operative pain has been a major medical deal.

a) Value of local administration of analgesic drugs: For the intramuscular injection of Naropein $\AA$ and opoids, the incidence of severe postoperative pain is reduced up to $29 \%$ and the incidence of catheter dislodgement is 5.7\% [2]. There is evidence that local anesthetic and opioid techniques are the most effective at relieving pain after major surgeries. Only regional anesthetic techniques, and preferably continuous techniques with local anesthesia, can result in a substantial reduction in pain response to surgical stress $[10,11]$.

b) Poor efficacy of spinal analgesia: Data on the use of epidural anesthesia in major orthopedic interventions are controversial. Although one study demonstrated a limited improvement in rehabilitation after knee replacement surgery, hospital stay was not improved [5].

c) Local analgesia in the area of surgical trauma, with minimal systemic side effects, appears to be an interesting challenger [12].

d) A preliminary series of 60 patients underwent local postoperative analgesia with a cellulose pad. We found that:-

- The pad implanted near the prosthesis did not pose any tolerance problems.

- A patient was readmitted on day 21 for leg ulcers. But the operative site was sterile and the compress was gone.

- Two-thirds of patients described postoperative pain rated 3 from the first day. 
- There was no difference between patients treated with conventional parietal infiltration and those who had the Naropein ${ }^{\circledR}$ pad.

e) We enjoyed a much simpler follow-up than perineural catheters we previously used in our service.

f) 8 out of 9 ambulatory patients were very satisfied of their local analgesia without any particular nursing care.

g) Systemic toxicity of local anesthetics: If safe anesthetic plasma concentrations are exceeded, signs of systemic toxicity appear. With increasing plasma levels, neurotoxic symptoms are the first to appear, followed by cardio toxicity that can be life threatening. Cardiac arrhythmias with cardiovascular collapse occur at levels approximately three times higher than those causing seizures. Allergic reaction to local anesthetics is very rare. Many of the reported reactions have a different underlying pathophysiological mechanism (systemic toxicity, vagal syncope) [13].

\section{Conclusion}

A series of 60 patients operated on a hip or knee prosthesis possible to get a first idea of the interest of Naropein ${ }^{\circledR}$ in situ. The use of a fibrillated cellulose pad does not pose a problem of tolerance. The subjective results on postoperative pain are at least equivalent to the intramuscular infiltration of the analgesic. Improvements are possible in the choice of the anesthetic and its combination with other molecules this could prolong his action.

\section{References}

1. Fletcher D, Fermanian C, Mardaye A, et al. A patient-based national survey on postoperative pain management in France reveals significant achievements and persistent challenges. Pain. 2008;137(2):441-451.

2. Dolin SJ, Cashman JN, Bland JM. Effectiveness of acute postoperative pain management: I. Evidence from published data. Br J Anaesth. 2002;89(3):409-423.

3. Bollish SJ, Collins CL, Kirking DM et al. Efficacy of patientcontrolled versus conventional analgesia for postoperative pain. Clin Pharm. 1985;4(1):48-52.
4. Aguirre J, Baulig B, Dora C et al. Continuous epicapsular ropivacaine $0.3 \%$ infusion after minimally invasive hip arthroplasty: a prospective, randomized, double-blinded, placebo-controlled study comparing continuous wound infusion with morphine patient-controlled analgesia. Anesth Analg. 2012;114(2):456-461.

5. Moiniche S, Mikkelsen S, Wetterslev J et al. A qualitative systematic review of incisional local anaesthesia for postoperative pain relief after abdominal operations. Br J Anaesth. 1998;81(3):377-383.

6. Capdevila X, Barthelet Y, Biboulet P et al. Effects of perioperative analgesic technique on the surgical outcome and duration of rehabilitation after major knee surgery. Anesthesiol. 1999;91(1):8-15.

7. Rawal N, Hylander J, Nydahl PA et al. Survey of postoperative analgesia following ambulatory surgery. Acta Anaesthesiol Scand. 1997;41(8):1017-1022.

8. Gunnar A, Sven B, Kurt K et al. Postoperative drainage of knee arthroplasty is not necessary: a randomized study of 90 patients. Acta Orthop Scand. 1998;69(5):475-478.

9. Williams Russo P, Sharrock NE, Steven BH et al. Randomized trial of epidural versus general anesthesia: Outcomes after primary total knee replacement. Clin Orthop Rel Res. 1996;331(331):199-208.

10. Kehlet H, Holte K. Effect of postoperative analgesia on surgical outcome. Br J Anaesth. 2001;87(1):62-72.

11. Kehlet H, Werner M, Perkins F. Balanced analgesia: what is it and what are its advantages in postoperative pain? Drugs. 1999;58(5):793-797.

12. Busch CA, Shore BJ, Bhandari R et al. Efficacy of periarticular multimodal drug injection in total knee arthroplasty. A randomized trial. J Bone Joint Surg Am. 2006;88(5):959-963.

13. Málek J, Ševčík P, Bejšovec D et al. Management of postoperative pain. Mladá Fronta. 2017.

\section{*Correspondence to:}

Dr. Dominique Persoons

Orthopedic surgeon, Hopital de Saverne, Cote de Saverne, France

E-mail - pers00ns@aol.com 